\title{
Target search on a dynamic DNA molecule
}

\author{
Thomas Schötz, ${ }^{1}$ Richard A. Neher, ${ }^{2}$ and Ulrich Gerland ${ }^{1, *}$ \\ ${ }^{1}$ Arnold Sommerfeld Center for Theoretical Physics and Center for Nano Science, University of Munich, \\ Theresienstrasse 37, D-80333 München, Germany \\ ${ }^{2}$ Max Planck Institute for Developmental Biology, Spemannstrasse 35, D-72076 Tübingen, Germany
}

(Received 21 November 2010; revised manuscript received 14 September 2011; published 17 November 2011)

\begin{abstract}
We study a protein-DNA target search model with explicit DNA dynamics applicable to in vitro experiments. We show that the DNA dynamics plays a crucial role for the effectiveness of protein "jumps" between sites distant along the DNA contour but close in three-dimensional space. A strongly binding protein that searches by one-dimensional sliding and jumping alone explores the search space less redundantly when the DNA dynamics is fast on the time scale of protein jumps than in the opposite "frozen DNA" limit. We characterize the crossover between these limits using simulations and scaling theory. We also rationalize the slow exploration in the frozen limit as a subtle interplay between long jumps and long trapping times of the protein in "islands" within random DNA configurations in solution.
\end{abstract}

DOI: 10.1103/PhysRevE.84.051911

PACS number(s): 87.15.H-, 87.14.gk, 82.37.-j

\section{INTRODUCTION}

The quantitative characteristics of proteins searching for their specific target sites on long DNA molecules have become a paradigmatic question of biological physics [1-4]. The question is of considerable biological interest since search processes of this type are key steps in cellular functions. For instance, in signal transduction, a protein belonging to the large class of transcription factors conveys an external signal and triggers the appropriate genetic response by binding to specific target sites on the genomic DNA. Similarly, restriction enzymes, used by bacteria to fight invading viruses, search for cleavage sites marked by specific DNA sequences. It is generally assumed that the target search mechanism has been optimized by evolution due to selective pressure for fast signaling and rapid responses in competitive environments. From the physics perspective, the protein-DNA target search is a complex but tractable stochastic process that combines basic aspects of Brownian motion, polymer physics, and information theory [5-16]. Experimentally, the search process can be probed on the single-molecule level in vitro [17] and even in vivo [18].

Early in vitro experiments [2] indicated that the association rate of lac repressor to its target site embedded in short pieces of DNA is faster than the diffusion limit, $k_{a}=4 \pi D b$, for a direct binding reaction with diffusion constant $D$ and reaction radius $b$. Inspired by Adam and Delbrück's idea that reduction of dimensionality is a generic way to enhance reaction rates [19], Richter and Eigen [3] interpreted these experiments with a two-step mechanism where three-dimensional (3D) diffusion and nonspecific association to DNA is followed by one-dimensional (1D) diffusive sliding into the target site. In a seminal series of papers [4], Berg, Winter, and von Hippel then established much of what is known today about the protein-DNA search kinetics. They experimentally varied the nonspecific binding strength via the ion concentration, identified an optimum where the search is fastest, and explained the behavior in a theoretical analysis.

\footnotetext{
*gerland@1mu.de
}

The existence of an optimum reflects a generic tradeoff in search processes for hidden targets [20]: A stochastic local search is exhaustive but redundant; interrupting the search by phases of rapid movement to new territory is a time investment that pays off by reducing the redundancy. The optimal fraction of time spent in each of the two "modes" depends on the statistical characteristics of the search mechanism. The simplest scenario, where proteins slide diffusively along the DNA, dissociate spontaneously, and randomly reattach at uncorrelated positions, leads to an optimum where, on average, only half of the proteins are bound somewhere on the DNA and the other half is in solution [4]. Physically, this is best understood [9] in terms of the typical dwell times of a protein in the sliding mode $\tau_{s}$ and in the dissociated state $\tau_{d}$. The latter should be regarded as a fixed parameter, set by cell size and composition, whereas $\tau_{s}$ can be adapted by molecular evolution of the DNA-binding domain of the protein (to adjust the nonspecific affinity). If $\tau_{s}<\tau_{d}$, the protein spends too little time searching, while if $\tau_{s}>\tau_{d}$, the search is too redundant; the search is fastest when they are equal.

However, in bacterial cells, well-studied transcription factors are bound to DNA $\gtrsim 90 \%$ of the time [5]. This fact has drawn attention to the "intersegment transfer" $[4,11,13,15]$ of proteins within the same DNA molecule, between sites close in space but distant along the contour. Potentially, this process can destroy the redundancy of the 1D search without the price of interrupting it by long excursions into the solvent. The term was introduced for proteins with two DNA-binding domains and refers to a process during which the protein never detaches from the DNA; a similar transfer but with a brief unbound period is referred to as "hopping" [4]. In both cases, the essential difference to the uncorrelated random reattachment discussed above is the correlated nature of the process: Transfer does not occur with equal probability to every site on the DNA but to "linked" sites. Here we simply refer to both processes as "jumping."

The interplay of protein sliding and jumping leads to intricate search dynamics. An analytical study [11] considered the effect of jumps using the fractional Fokker-Planck equation [21], which assumes that consecutive jumps are uncorrelated, i.e., that the DNA configuration randomizes 


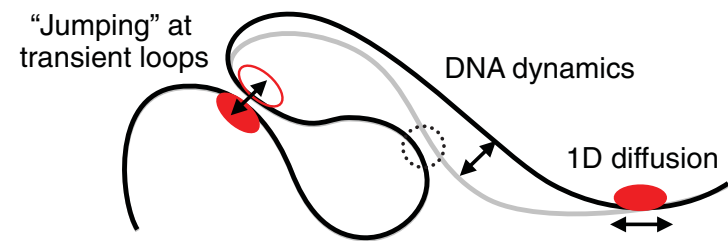

FIG. 1. (Color online) Illustration of the target search by sliding (1D diffusion) and jumping on a dynamic polymer.

between two jumps. In contrast, a numerical study of sliding and jumping on a random but frozen contour [22] showed that correlations between jumps drastically alter the dynamics, leading to "paradoxical" quasidiffusive behavior instead of superdiffusion along the contour. Specifically, the distribution of the protein on the DNA exhibits characteristic heavy tails even though its width increases only diffusively. These findings, and the fact that the dynamics of real DNA is neither frozen nor annealed over the relevant range of microsecond to second time scales [4], call for an analysis of target search on a dynamic DNA; see Fig. 1. Here we characterize the crossover between the frozen and the annealed regime using simulations and scaling theory. We then study the mechanism whereby correlated jumps create the paradoxical behavior in the frozen limit.

\section{MODEL}

To make the problem tractable, we describe the DNA contour as a path of $L$ segments on a simple cubic lattice and generate its conformational dynamics with a kinetic Monte Carlo scheme based on a generalized Verdier-Stockmayer move set [23] with moves for kinks, chain ends, and crankshafts; see Fig. 2. These moves, carried out at rate $k_{\mathrm{D}}$, implement Rouse dynamics on a lattice for an ideal chain (no self-avoidance). That crankshaft turns are necessary to obtain Rouse dynamics becomes intuitively clear if one considers
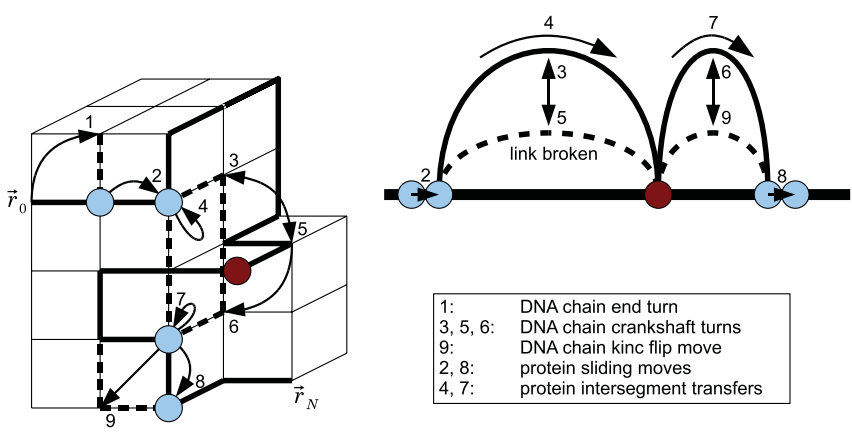

FIG. 2. (Color online) Illustration of the move set of our kinetic Monte Carlo scheme. (left) The DNA chain is represented by a path on a cubic lattice. The protein is represented by a point particle that moves at rate $k_{\mathrm{p}}$, either by randomly sliding along the chain contour or by jumping to another segment of the chain at the same position. (right) The link diagram representation has the DNA contour stretched out to a line and indicates possible jumps by arcs. Links can be created or destroyed by the Rouse dynamics of the DNA, which is implemented with a generalized Verdier-Stockmayer move set allowing for kink flips, turns at the chain end, and crankshaft moves. Each move is carried out at the rate $k_{\mathrm{D}}$. a planar DNA conformation as the initial state: without the crankshaft move, only the ends of the contour can move out of the plane, which would lead to an equilibration time slower than the $L^{2}$ scaling in the Rouse model.

We describe a protein as a point particle on the lattice, which diffuses along the DNA contour at rate $k_{\mathrm{p}}$. If another DNA segment passes through the same point, the protein can randomly jump to it (at the same rate $k_{\mathrm{p}}$, for simplicity). The dimensionless kinetic ratio

$$
k=k_{\mathrm{D}} / k_{\mathrm{p}}
$$

is an important parameter of our model that measures the time scale of the protein kinetics relative to the DNA kinetics (see further below for a rough experimental estimate). We focus on the limit of strong DNA binding without explicit 3D diffusion of the protein (although jumps may involve 3D diffusion, as discussed above). As initial condition, we use a random DNA configuration with the protein on the central segment. Clearly, the configuration of the DNA inside a bacterial cell is not random due to genome packaging and confinement, but a random configuration is an interesting starting point for the exploration of the physical principles and mimics the situation of in vitro experiments.

\section{RESULTS}

\section{A. Transport}

To characterize how a protein explores the search space, we study the time evolution of its probability distribution $P(s, t)$ along the DNA contour $(0 \leqslant s \leqslant L)$. Figure 3 plots its width $\Lambda(t)$, defined as the interquartile range $\Lambda=I^{-1}\left(\frac{3}{4}\right)-$ $I^{-1}\left(\frac{1}{4}\right)$ of the cumulative distribution $I(y)=\int_{0}^{y} d s P(s, t)$, for different kinetic ratios $k$. We obtain $P(s, t)$ by averaging over $\geqslant 10^{3}$ simulations, with $L=5000$ and different initial DNA configurations. In the "quenched limit" $k \rightarrow 0$ (squares), the protein moves on a frozen contour, and the width grows quasidiffusively with time, $\Lambda \sim t^{1 / 2}$, despite the long-range jumps along the contour and a heavy tail of the distribution $P(s, t)$ at fixed $t$ [22]. In the opposite "annealed limit" $k \rightarrow \infty$ (crosses, obtained by randomly drawing a new DNA configuration after each jump), the distribution initially spreads superdiffusively along the contour, $\Lambda \sim t^{\alpha}$ (here $\alpha \approx 1.7$ ). The width saturates at $\Lambda \rightarrow L / 2$ as the protein explores the

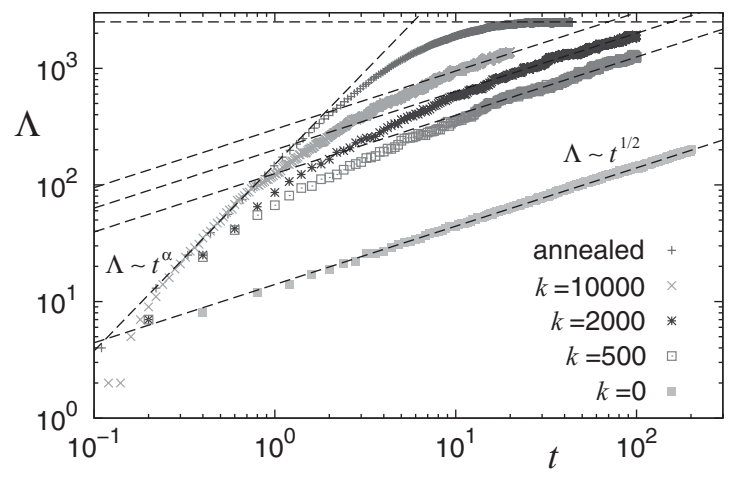

FIG. 3. Time evolution of the width $\Lambda$ of the protein distribution $P(s, t)$ for different kinetic ratios $k=k_{\mathrm{D}} / k_{\mathrm{p}}$. A crossover from superdiffusive to quasidiffusive dynamics occurs for finite $k$. 

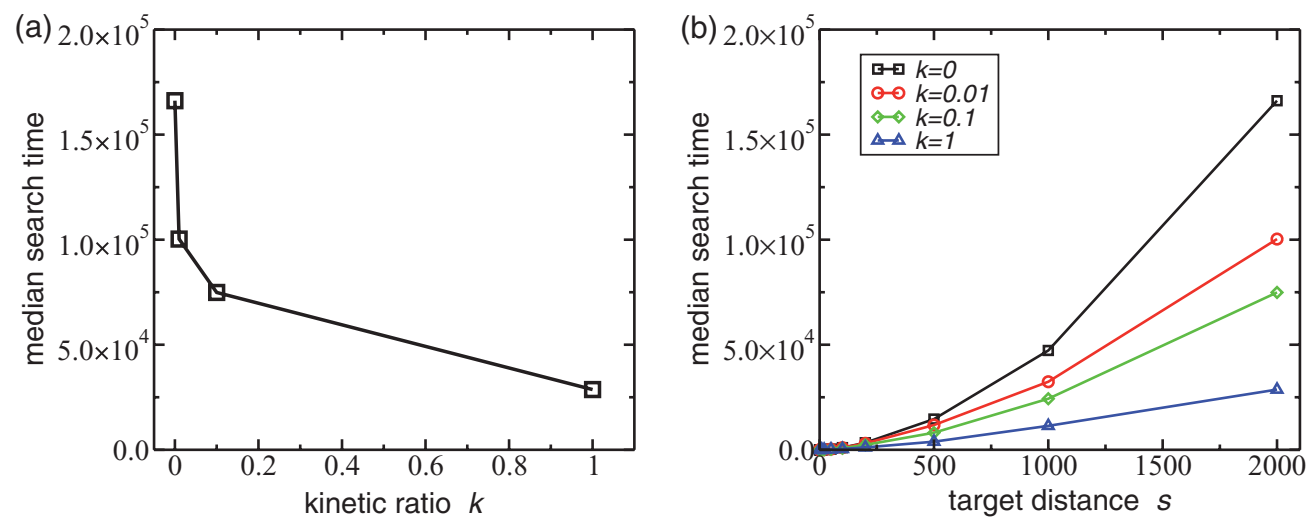

FIG. 4. (Color online) Simulation results for the target search on the dynamic DNA chain (of length $L=5000$ ). The protein was initially placed in the center of the chain and the time was measured until it first arrived at a target site placed a distance $s$ away. These simulations were performed 1000 times, using different initial polymer configurations, to determine the median of the search time, which represents a typical search time. Both $s$ and the kinetic ratio $k=k_{\mathrm{D}} / k_{\mathrm{p}}$ of DNA to protein moves were varied. The simulation of the target search on the dynamic chain is computationally expensive, which limits our range of $k$ values. (a) The typical search time to a target site at distance $s=2000$ as a function of the kinetic ratio $k$. A substantial increase of the search time with slowing DNA kinetics is apparent. (b) The typical search time plotted against the distance of the target site for the different kinetic ratios. The dependence of the search time on the distance $s$ becomes weaker as $k$ is increased (however, a significant dependence on $s$ remains for all our $k$ values).

entire DNA. In the regime of intermediate $k$, which is relevant in most experimental situations, $\Lambda(t)$ displays a crossover from superdiffusive to quasidiffusive scaling. The curves for different $k$ show that the crossover time scale $\tau_{c}$ increases with $k$.

For large $k$, the connectivity of the DNA meshwork on which the protein moves changes rapidly, such that successive jumps are uncorrelated (they occur on different link sets). One can then describe the dynamics by the average jump probability $P\left(s, s^{\prime}\right)$ from site $s$ to site $s^{\prime}$, which is physically determined by the DNA looping probability. For an ideal chain, this probability decays as $\left|s-s^{\prime}\right|^{-3 / 2}$ for large loops before it is cut off by the finite DNA length. When successive jump lengths are independently drawn from this distribution, the typical distance $\Lambda$ from the initial position is dominated by the largest jump, which grows with the number of jumps $(\sim t)$ as $\Lambda(t) \sim t^{2}$ [24]. Indeed, our numerical exponent $\alpha$ approaches 2 at large $L$ (data not shown). However, what does the transport $\Lambda(t)$ imply for the target search process?

\section{B. Search time}

Without a guiding "funnel," no search process can be faster than linear exploration. A faster than linear $\Lambda(t)$ leads to a "sloppy search" [11] where patches dispersed over the entire contour are explored before the target is located. This is precisely what is required to break the redundancy of $1 \mathrm{D}$ diffusion, suggesting that jumping is an effective mechanism that could replace $3 \mathrm{D}$ diffusion in the annealed regime. On the other hand, we expect that jumping is ineffective in the frozen limit, as it leads only to quasidiffusive spreading along the DNA. To study the target search on dynamic DNA explicitly, we performed simulations with a target site placed at different distances from the initial protein position. Figure 4(a) shows that the search indeed takes increasingly longer as the DNA dynamics is slowed. Figure 4(b) shows that the strong dependence of the search time on the initial distance to the target (at $k=0$ ) becomes weaker as $k$ is increased; see caption for details.

It will require single-molecule experiments of the type of [18] (but under controlled in vitro conditions) to find out which regime of $k$ values is biologically most relevant. However, a rough estimate, based on the experimental relaxation time of $\tau=30 \mathrm{~s}$ for the contour of a $L=43 \mu \mathrm{m}$ DNA fragment and the experimental scaling law $\tau \sim L^{1.65}$ [25], indicates that on the millisecond time scale of protein jumps [4], only short DNA segments will be equilibrated. We therefore expect that neither the annealed nor the frozen limit but the crossover regime will be most relevant experimentally.

\section{Scaling of the crossover}

To understand the physics of the crossover regime within our model, we apply a scaling argument to the interplay of DNA and protein dynamics: A DNA segment of length $\ell$ equilibrates on a time scale $\tau \sim \ell^{2}$ (Rouse dynamics). Within a time $\tau$ after a protein docks onto the DNA and starts exploring, it typically visits a DNA stretch $\Lambda(\tau)$. During this time, a DNA segment of size $\ell \sim\left(k_{\mathrm{D}} \tau\right)^{1 / 2}$ equilibrates. Superdiffusive protein transport results as long as $\Lambda(\tau)<\ell$; however, the fast growing $\Lambda(t) \sim\left(k_{\mathrm{p}} t\right)^{\alpha}$ quickly outruns the "equilibration blob," and the passing point marks the crossover to the quasidiffusive regime. With $\alpha=2$, this crossover time scale $t_{c}$ then depends on the kinetic ratio $k$ as $k_{\mathrm{p}} t_{c} \sim k^{1 / 3}$. Our simulations cannot explore a wide range of $k$ values due to computational cost and do not allow a precise determination of this scaling (however, the scaling exponent that best describes our limited data deviates only by 0.08 from the expected value of 1/3; see Fig. 5). The small numerical value of the exponent leads to a broad crossover as a function of $k$, again suggesting that neither the annealed nor the frozen limit is experimentally attainable. 


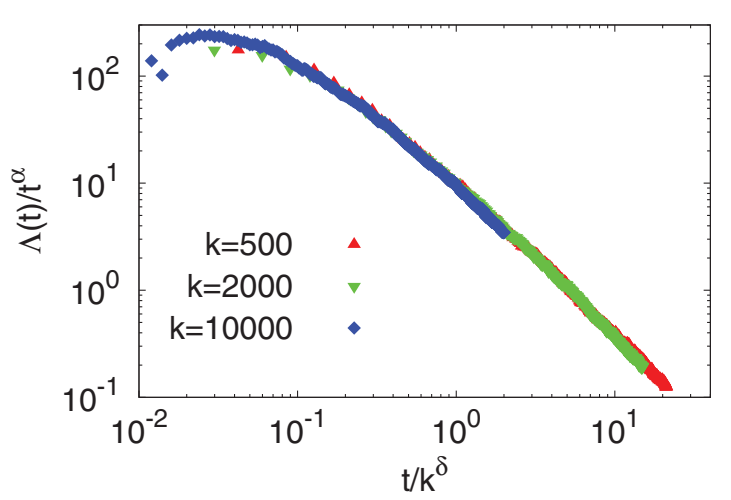

FIG. 5. (Color online) Data collapse to extract an estimate of the scaling behavior of the crossover between superdiffusive and quasidiffusive dynamics. The three curves from Fig. 3 with different finite kinetic ratios $k$ can be collapsed onto each other by rescaling the axes (here we have used the asymptotic value of 2 for the exponent $\alpha)$. The best collapse is obtained when the time is rescaled as $t / k^{\delta}$ with $\delta$ around 0.25 . This exponent deviates from the theoretically expected value of $1 / 3$; however, the deviation is not significant given the finite size effect of our simulations.

\section{Quenched limit}

To obtain a better understanding of the mechanism responsible for the slowdown of the search, we focus on the quenched limit. When first reported [22], the quasidiffusive transport was attributed to correlation effects. However, what is the nature of these correlations, and how do they render the long-range jumping process quasidiffusive? We distinguish two types of correlations, which we refer to as temporal and spatial. For this distinction, it is useful to picture the DNA configuration in the link diagram representation of Fig. 2. On static DNA, a protein can use the same links multiple times, leading to temporal correlations. Additionally, the positions of different links are spatially correlated since an existing link strongly

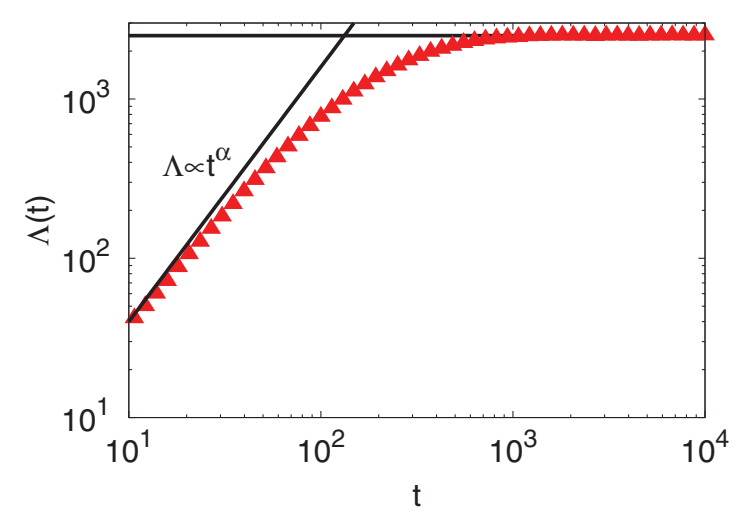

FIG. 6. (Color online) Kinetic Monte Carlo simulation of the protein dynamics on reshuffled link sets. The data are obtained from simulations using the actual link set of a random DNA configuration and then randomly reshuffling the positions of these links (while conserving the length of each link). The protein dynamics is simulated on this reshuffled but temporally fixed link set. Finally, the average dynamics of the width $\Lambda(t)$ is obtained by averaging over many initial DNA configurations (each randomly drawn). The dynamics of $\Lambda(t)$ is superdiffusive, showing that the temporal correlations are not sufficient to produce the quasidiffusive behavior (see main text).

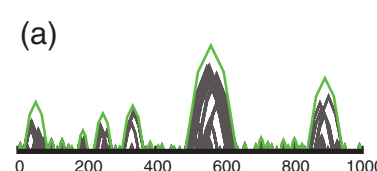

(b)
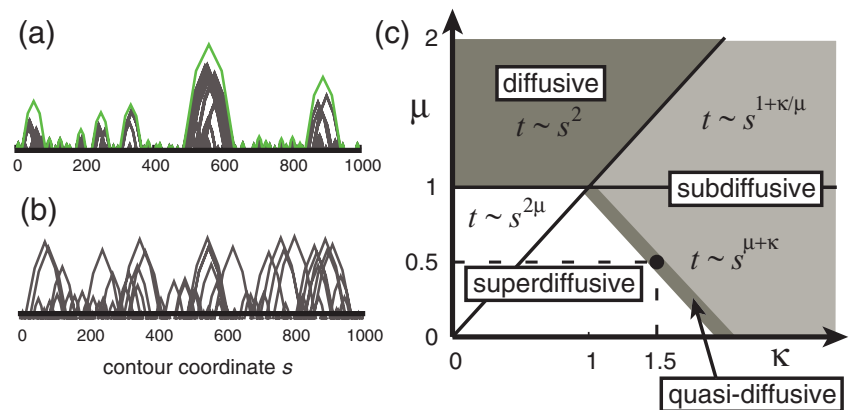

FIG. 7. (Color online) (a) The link diagram for a typical DNA conformation is separable into islands (top green line). (b) Random reshuffling of the same links destroys the islands. (c) A toy model for transport on the island structure leads to the dynamical phase diagram, which explains the quasidiffusive regime as a cancellation of the effect of traps and long-range jumps.

enhances the probability to find another link nearby (e.g., a loop in the DNA favors further contacts within the loop). To separate the effect of temporal and spatial correlations, we destroy the latter by choosing a new random starting point for each link while conserving its arc length $\left|s-s^{\prime}\right|$. The protein transport on such reshuffled link sets is superdiffusive, as revealed by simulations shown in Fig. 6. Hence temporal correlations alone are not sufficient to cause the quasidiffusive behavior. A simple argument makes this plausible: If the region visited by the protein grows superdiffusively as $\Lambda(t) \sim t^{2}$, the protein visits only a fraction $\sim 1 / t$ of the sites within $\Lambda$. Since it sees each site $\mathcal{O}(1)$ times, it mostly uses novel links, and the persistence of links is unimportant.

\section{E. Islands}

A striking consequence of the spatial correlations is revealed in Fig. 7(a), where all links in a typical DNA configuration are depicted as arcs. The arcs cluster into "islands" with many internal links but no links between islands.

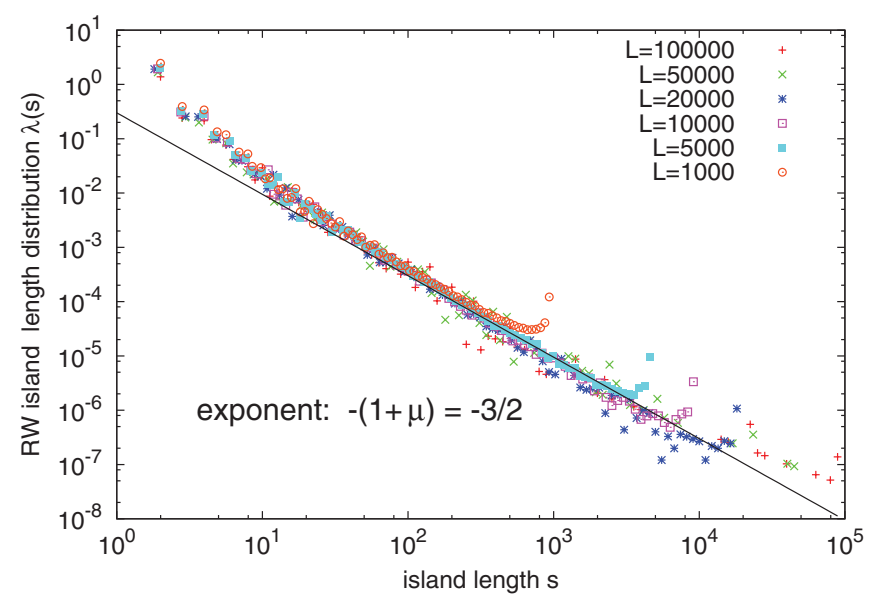

FIG. 8. (Color online) Distribution of islands sizes. The distribution was obtained by generating random DNA conformations of length $L$ and picking a single island from each link diagram at random. The distribution displays the same power law decay as the distribution of link lengths. 


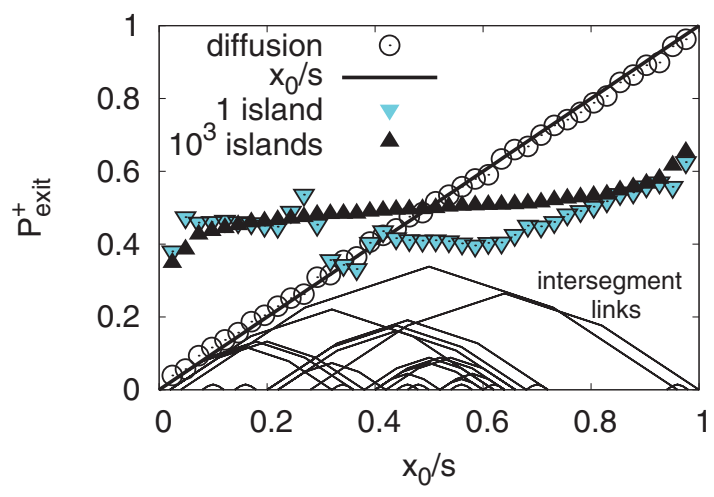

FIG. 9. (Color online) Exit probability from an island to the right as a function of the starting position $x_{0}$ (normalized here by the island size $s$ ). The exit probability is shown both for a single randomly chosen island (blue downward triangles) with the link configuration indicated by the link diagram in the bottom and averaged over an ensemble of 1000 islands of the same size (black triangles). For comparison, the case of pure diffusion (no links), where the exit probability depends linearly on the starting position, is also shown (the solid line shows the analytical dependence, while the circles indicate simulation data, which were obtained as a control using the same simulation code as for the islands). It is evident that the probability of exiting an island on a given side depends only weakly on the initial position, at least in the core of the island. This justifies our coarse grained hopping model in island space.

These islands disappear when the same links are randomly placed on the DNA; see Fig. 7(b). Intuitively, it is clear that the existence of islands slows the exploration of the DNA since the protein can move from one island to another only by sliding. In fact, if the islands had a well-defined typical size $\bar{s}$, the protein dynamics would be diffusive on long scales $s \gg \bar{s}$. However, the problem is more intricate since the distribution of island sizes has the same heavy tail $p(s) \sim s^{-3 / 2}$ as the link length distribution; see Fig. 8. Nevertheless, the existence of islands is a crucial clue; we show below that it leads to a dynamics that can be described by a 1D transport model with traps and long-distance jumps. To this end, we first note two essential transport properties of islands: (i) Due to the internal links, the position of a protein is rapidly randomized within an island, such that for most starting positions within an island, it leaves the island with nearly the same probability to each side; see Fig. 9. (ii) The typical trapping time within an island scales as $\tau \sim s^{3 / 2}$ with the island size; see Fig. 10 .

Given these properties, we consider protein transport on an array of islands with sizes $s_{i}$ drawn from the distribution $p(s)$. Each island has an associated trapping time $\tau_{i}\left(s_{i}\right)$. It will be instructive to allow for adjustable exponents $\mu$ and $\kappa$ in the scaling behavior, $p(s) \sim s^{-1-\mu}$ and $\tau \sim s^{\kappa}$. Combining these relations, we obtain a distribution of trapping times $w(\tau) \sim$ $\tau^{-1-\mu / \kappa}$ since $w(\tau) d \tau=p(s) d s$. The transport behavior of the protein in island space is then determined by the ratio of the exponents: Using the first passage time calculus [26], the typical time needed to move over $n$ islands is

$$
T \sim n \sum_{i=1}^{n} \tau_{i} \sim \begin{cases}n^{1+\frac{\kappa}{\mu}} & \text { for } \kappa>\mu \\ n^{2} & \text { for } \kappa<\mu\end{cases}
$$

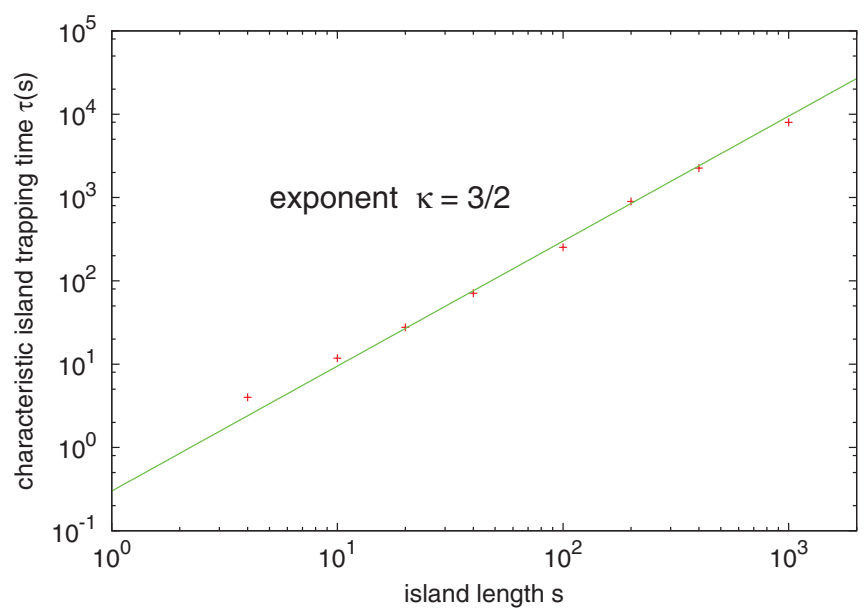

FIG. 10. (Color online) Average trapping time of a protein within an island as a function of island size $s$. Each data point is obtained as an average over many simulations where a protein is initialized in the center of an island of size $s$ within a randomly drawn DNA configuration, and the time until it exits from the island is recorded. This island size dependent characteristic trapping time scales as $\tau(s) \sim s^{3 / 2}$.

with the sum dominated by the largest term for the case $\kappa>\mu$ while a typical trapping time exists for $\kappa<\mu$. To map the dynamics in island space back onto the DNA, note that the total DNA length $S$ of $n$ islands scales as

$$
S(n) \sim\left\{\begin{array}{ll}
n^{1 / \mu} & \text { for } \mu<1 \\
n & \text { for } \mu>1
\end{array},\right.
$$

as $S$ is dominated by the largest island for $\mu<1$. Combining (2) and (3) yields the transport behavior along the DNA, i.e., the typical time to travel a given distance. Figure 7(c) shows the phase diagram spanned by the exponents $\mu$ and $\kappa$. It exhibits four different regimes. For $\mu>1$, the distribution of island sizes has a well-defined mean and no superdiffusion can occur, but subdiffusive dynamics results when the trapping time distribution has a sufficiently heavy tail $(\mu<\kappa)$. If $\mu<1$, the dynamics is superdiffusive unless long trapping times in islands compensate for long jumps. In particular, $t \sim s^{\mu+\kappa}$ for $\mu<\kappa$, which includes the case of interest here, where the two exponents precisely add up to 2 , rationalizing quasidiffusion in the quenched limit. Within our more general island model, a whole line of points exists where the dynamics is quasidiffusive. In contrast, for the protein transport on the DNA contour, $\mu$ and $\kappa$ are not independent since they are both related to the statistics of the network topologies created by the DNA conformations. Why this leads to $\mu+\kappa=2$ remains to be understood.

\section{CONCLUSION}

We analyzed the transport and search of proteins on a dynamic DNA contour. We showed that the highly correlated nature of the protein dynamics persists over a broad range of our dimensionless dynamic parameter $k=k_{\mathrm{D}} / k_{\mathrm{p}}$ and significantly slows down the search process. Our findings imply that under the in vitro conditions of our model, protein jumping is effective as a mechanism to destroy the redundancy 
of a diffusive 1D search only if the DNA dynamics is sufficiently fast compared to the time scale between protein jumps or if many proteins search in parallel. Of course, the in vivo situation is complicated by many additional factors, such as the nonrandom conformation and the confinement of the DNA. We also found that the "paradoxical" quasidiffusive dynamics in the quenched limit [22] can be viewed as a subtle cancellation of the effect of traps and long-distance jumps. The interplay between traps, jumps, and memory in 1D transport is an intricate problem in statistical mechanics [27,28]. The
protein-DNA system naturally displays a nontrivial interplay and surprisingly is tuned to a critical point in our dynamical phase diagram.

\section{ACKNOWLEDGMENTS}

We acknowledge useful discussions with Thomas Franosch, Yariv Kafri, Joseph Klafter, Ralf Metzler, and Igor Sokolov and funding by the DFG via the excellence cluster Nano Initiative Munich (NIM).
[1] S. E. Halford and J. F. Marko, Nucleic Acids Res. 32, 3040 (2004).

[2] A. D. Riggs, M. S. Bourgeois, and M. Cohn, J. Mol. Biol. 53, 401 (1970).

[3] P. H. Richter and M. Eigen, Biophys. Chem. 2, 255 (1974).

[4] O. G. Berg, R. B. Winter, and P. H. von Hippel, Biochemistry 20, 6929 (1981); R. B. Winter and P. H. von Hippel, ibid. 20, 6948 (1981); R. B. Winter, O. G. Berg, and P. H. von Hippel, ibid. 20, 6961 (1981).

[5] G. D. Stormo and D. S. Fields, Trends Biochem. Sci. 23, 109 (1998).

[6] U. Gerland, J. D. Moroz, and T. Hwa, Proc. Natl. Acad. Sci. USA 99, 12015 (2002).

[7] R. F. Bruinsma, Phys. A 313, 211 (2002).

[8] D. Brockmann and T. Geisel, Phys. Rev. Lett. 91, 048303 (2003).

[9] M. Slutsky and L. A. Mirny, Biophys. J. 87, 4021 (2004).

[10] M. Coppey, O. Bénichou, R. Voituriez, and M. Moreau, Biophys. J. 87, 1640 (2004)

[11] M. A. Lomholt, T. Ambjörnsson, and R. Metzler, Phys. Rev. Lett. 95, 260603 (2005).

[12] T. Hu, A. Y. Grosberg, and B. I. Shklovskii, Biophys J. 90, 2731 (2006).

[13] T. Hu and B. I. Shklovskii, Phys. Rev. E 76, 051909 (2007).

[14] Z. Wunderlich and L. A. Mirny, Nucleic Acids Res. 36, 3570 (2008).
[15] M. Sheinman and Y. Kafri, Phys. Biol. 6, 016003 (2009).

[16] N. Geisel and U. Gerland, Biophys. J. 101, 1569 (2011).

[17] B. van den Broek, M. A. Lomholt, S.-M. J. Kalisch, R. Metzler, and G. J. L. Wuite, Proc. Natl. Acad. Sci. USA 105, 15738 (2008).

[18] J. Elf, G.-W. Li, and X. S. Xie, Science 316, 1191 (2007).

[19] G. Adam and M. Delbrück, in Structural Chemistry and Molecular Biology, edited by A. Rich and N. Davidson (Freeman, San Francisco, 1968), p. 198.

[20] O. Bénichou, M. Coppey, M. Moreau, P.-H. Suet, and R. Voituriez, Phys. Rev. Lett. 94, 198101 (2005).

[21] R. Metzler and J. Klafter, Phys. Rep. 339, 1 (2000).

[22] I. M. Sokolov, J. Mai, and A. Blumen, Phys. Rev. Lett. 79, 857 (1997).

[23] D. P. Landau and K. Binder, A Guide to Monte Carlo Simulations in Statistical Physics (Cambridge University Press, Cambridge, 2000).

[24] J.-P. Bouchaud and A. Georges, Phys. Rep. 195, 127 (1990).

[25] T. T. Perkins, S. R. Quake, D. E. Smith, and S. Chu, Science 264, 822 (1994).

[26] C. Gardiner, Handbook of Stochastic Methods (Springer, Berlin, 2004).

[27] G. Zumofen and J. Klafter, Phys. Rev. E 51, 1818 (1995).

[28] N. Kumar, U. Harbola, and K. Lindenberg, Phys. Rev. E 82, 021101 (2010). 